\title{
Adverse prognostic value of PD-L1 expression in primary resected pulmonary squamous cell carcinomas and paired mediastinal lymph node metastases
}

\author{
Manuel D Keller ${ }^{1}$, Christina Neppl ${ }^{1}$, Yasin Irmak $^{1}$, Sean R Hall ${ }^{2,3}$, Ralph A Schmid ${ }^{2,3}$, \\ Rupert Langer $^{1}$ and Sabina Berezowska ${ }^{1}$ \\ ${ }^{1}$ Institute of Pathology, University of Bern, Bern, Switzerland; ${ }^{2}$ Division of General Thoracic Surgery, \\ Inselspital University Hospital Bern, Bern, Switzerland and ${ }^{3}$ Department of Clinical Research, University of \\ Bern, Bern, Switzerland
}

\begin{abstract}
Immunohistochemical assessment of programmed cell death (PD)-ligand 1 (PD-L1) expression in lung cancer in the context of therapeutically targeting the PD1/PD-L1 axis is still controversially discussed. This includes the comparability of antibody clones, prognostic value, and discrepancies between primary tumors and metastases. We assessed tumoral PD-L1 expression using clones E1L3N and SP142 in 372 primary resected pulmonary squamous cell carcinomas, including 40 paired N2 lymph node metastases, in relation with clinico-pathological parameters. PD-L1 expression was negative $(<1 \%)$ in $163 / 372(44 \%$, E1L3N) or $231 / 370$ patients $(62 \%$, SP142). Positivity of $1-<50 \%$ was observed in $135(36 \%$, E1L3N) or 92 patients $(25 \%$, SP142) and $\geq 50 \%$ in $74(20 \%$, E1L3N) or 47 patients (13\%, SP142). PD-L1 staining correlated significantly between both antibodies ( $r=0.781$; $P<0.001)$. Scores correlated significantly between full-slide sections $(N=40)$ and tissue microarrays, and between primaries and $\mathrm{N} 2$ metastases $\left(P<0.001\right.$ all). $\mathrm{CD}^{+}$tumor infiltrating lymphocyte counts positively correlated with PD-L1 expression $(P<0.001)$. PD-L1 $\geq 50 \%$ showed the best prognostic discrimination using the split-sample validation method. It was associated with shorter disease-specific survival in the observation group (E1L3N: $P=0.035$, SP142: $P=0.002$ ) and validation group (E1L3N: $P=0.024$, SP142: $P=0.101$ ) and shorter time to recurrence (observation group: E1L3N: $P=0.056$, SP142: $P<0.001$; validation group: E1L3N: $P=0.036$, SP142: $P=0.247$ ). Multivariate analysis showed that PD-L1 expression $\geq 50 \%$ determined by clone E1L3N was an independent prognostic factor in the observation group regarding disease-specific survival $(\mathrm{HR}=2.768 ; 95 \%$ $\mathrm{Cl}=1.149-6.666 ; P=0.023)$ and time to recurrence $(\mathrm{HR}=2.164 ; 95 \% \mathrm{Cl}=1.056-4.436 ; P=0.035)$ and in the validation group (disease-specific survival: $\mathrm{HR}=1.978 ; 95 \% \mathrm{Cl}=0.928-4.214 ; P=0.077$ and time to recurrence: $\mathrm{HR}=1.571 ; 95 \% \mathrm{Cl}=0.838-2.944 ; P=0.159)$. High PD-L1 expression was associated with adverse prognosis in pulmonary squamous cell carcinoma. Clone E1L3N was more sensitive than SP142 and superior regarding prognostication. PD-L1 expression correlated significantly between primary tumor and N2 metastases, rendering mediastinal lymph node metastases adequate for immunohistochemical assessment.

Modern Pathology (2018) 31, 101-110; doi:10.1038/modpathol.2017.111; published online 8 September 2017
\end{abstract}

Lung cancer, comprising non-small cell lung cancer (NSCLC) as the largest subgroup, belongs to the most frequent malignancies worldwide. ${ }^{1}$ In advanced stages, radiation and chemotherapy render unsatisfactory clinical results, making it the leading cause of cancer-related deaths in the Western world. ${ }^{1,2}$ In

Correspondence: Dr S Berezowska, MD, Institute of Pathology, University of Bern, Murtenstrasse 31, Bern 3008, Switzerland.

E-mail: sabina.berezowska@pathology.unibe.ch

Received 29 April 2017; revised 19 July 2017; accepted 19 July 2017; published online 8 September 2017 contrast to adenocarcinomas, for squamous cell carcinomas no targeted therapy is available to date. ${ }^{3}$

Targeting the programmed death protein 1 (PD1)/ programmed death-ligand 1 (PD-L1) interaction proved impressively efficacious in NSCLC, and rapidly translated into clinically approved therapeutics. ${ }^{2,4-8}$ PD-L1 on the tumor cell binds to PD1 on the effector $\mathrm{T}$ cell, thereby protecting the tumor by shutting down the T-cell response. PD1/PD-L1 inhibitors block this immunoescape, leading to elimination of the malignant cells through 
reinvigorated immune surveillance.9,10 Predictive markers are needed, as only around $20 \%$ of unselected NSCLC patients respond to therapy and immunotherapy has already arrived in the first-line setting, competing with effective standard therapies. ${ }^{11,12}$

Immunohistochemical staining for PD-L1 is technically well applicable in histopathology laboratories, but presents many uncertainties to date. Its prognostic but also its predictive role, or the congruence between the expression in primary tumors and metastases are controversial. Nevertheless, PD-L1 expression on the tumor cells is still regarded the best single predictive biomarker for anti-PD1/PD-L1 therapy in NSCLC. ${ }^{9,13,14}$

We aimed to validate the prognostic value and heterogeneity of PD-L1 expression in primary tumors and lymph node metastases in squamous cell carcinomas of the lung in a large Western cohort.

\section{Materials and methods}

\section{Patient Cohort}

We retrospectively analyzed 378 consecutive patients with primary resected pulmonary squamous cell carcinomas, diagnosed at the Institute of Pathology, University of Bern, January 2000-December 2013, without previous or concomitant diagnosis of squamous cell carcinomas of other organs to reliably exclude metastatic lung disease. The study was conducted according to the REMARK guidelines, ${ }^{15}$ and was approved by the Cantonal Ethics Commission of the Canton of Bern (KEK 200/14), which waived the requirement for written informed consent. Initial inclusion criteria were matched by 402 patients according to the pathology files. ${ }^{16}$ Re-evaluation of clinical files and histology led to exclusion of 24 additional patients, 13 due to neoadjuvant treatment not mentioned in the pathology files, 3 due to previous squamous cell carcinomas of other organs, 1 due to lack of sufficient available tissue, and 7 due to TTF1 positivity in the tumors, suggestive of adenocarcinoma. ${ }^{17}$ All tumors were re-staged according to the UICC 2009 7th edition TNM classification (Table 1). ${ }^{18}$

Survival data were available for 278 patients. Time to recurrence was measured from the time of resection to loco-regional or metastatic recurrence or disease-specific death. Mean time to recurrence in the total cohort was 105 months (95\% CI=93116 months). Disease-specific survival was measured from the time of resection to disease-specific death. Patients who died due to unrelated causes or secondary malignancies were censored at the time of death. Mean disease-specific survival in the total cohort was 139 months (95\% CI=126-151 months) and mean overall survival was 83 months $(95 \%$ $\mathrm{CI}=74-92$ months). Of note, 100/159 (63\%) deaths were unrelated to pulmonary squamous cell carcinoma (eg, heart attack, secondary lung adenocarcinoma) and 59/159 (37\%) were disease-specific deaths.

\section{Tissue Microarray and Full-Slide Analysis}

A next-generation tissue microarray, with digital annotation of scanned slides and automatic transferal of the punches was constructed from formalinfixed and paraffin-embedded tissue blocks archived in the Institute of Pathology, University Bern, Switzerland, as described before. ${ }^{16,19}$ One representative tissue block was selected for each tumor after reviewing all available slides per case (hematoxylin and eosin stained), and eight tumor cores (diameter $0.6 \mathrm{~mm}$ ) were randomly selected from the block by placing digital annotations on the scanned slide. It has been shown before that one block/tumor is sufficient to represent the spatial heterogeneity of PD-L1 expression of the tumor. ${ }^{20}$ The eight cores/ tumor were placed on four different tissue microarray blocks to exclude technical staining bias.

Additionally, full tissue sections were used in the pN2 subcohort for comparison between tissue microarray cores and full tissue sections and between primaries and lymph node metastases.

\section{Immunohistochemistry}

Immunohistochemical staining was performed using an automated immunostainer (Bond III, Leica Biosystems, Muttenz, Switzerland) using the following antibodies: anti-CD3 (Abcam Cambridge, UK, clone SP7, Tris-buffer, $95^{\circ} \mathrm{C} 30 \mathrm{~min}$, 1:400), anti-CD8 (Dako, clone C8/144B, Tris-buffer, $95^{\circ} \mathrm{C} 20 \mathrm{~min}$, $1: 100$ ), as described before. ${ }^{19}$ The anti-TTF1 clone 8G7G3/1 (Cell Marque, Rocklin, CA, USA) was used at a dilution of 1:400 after antigen retrieval performed with Tris-EDTA, pH 9 for $30 \mathrm{~min}$ at $95^{\circ} \mathrm{C}$. PD-L1 immunohistochemistry was performed using laboratory-developed tests using clone SP142 (Spring Bioscience, Pleasanton, CA, USA) and clone E1L3N (Cell Signaling Technology, Danvers, MA, USA) at a dilution of 1:400 each, as described before. ${ }^{21}$ Both PD-L1 clones have been validated for specificity. ${ }^{21-23}$ Deparaffinized sections were rehydrated in Dewax dilution (Leica Biosystems) for $20 \mathrm{~min}$, and antigen retrieval was performed with Tris-HCl, pH 9 for $40 \mathrm{~min}$ at $95^{\circ} \mathrm{C}$. Endogenous peroxidase activity was blocked with $\mathrm{H}_{2} \mathrm{O}_{2}$ solution (Leica Biosystems). Samples were incubated with primary antibodies at room temperature for $15 \mathrm{~min}$, followed by incubation with the secondary antibody using the Bond Polymer Refine Kit with 3-3'diaminobenzidine-DAB as chromogen (Leica Biosystems), counterstained with hematoxylin and mounted in Aquatex (Merck, Darmstadt, Germany).

PD-L1 expression was assessed as proportion of tumor cells showing membranous staining of any intensity (tumor proportion score) by a pathologist 
Table 1 Clinico-pathological characteristics of the patient cohort in relation with PD-L1 expression (clone E1L3N)

\begin{tabular}{|c|c|c|c|c|c|c|}
\hline \multirow{2}{*}{ Factors } & \multicolumn{2}{|c|}{ Number of patients } & \multicolumn{3}{|c|}{ PD-L1 expression } & \multirow{2}{*}{ P-value } \\
\hline & Total cohort & PD-L1 evaluation & $<1 \%$ & $1-<50 \%$ & $\geq 50 \%$ & \\
\hline Total & 378 & 372 & $163(44 \%)$ & $135(36 \%)$ & $74(20 \%)$ & \\
\hline \multicolumn{7}{|l|}{ Gender } \\
\hline Male & $320(85 \%)$ & 315 & $144(46 \%)$ & $111(35 \%)$ & $60(19 \%)$ & 0.217 \\
\hline Female & $58(15 \%)$ & 57 & $19(33 \%)$ & $24(42 \%)$ & $14(25 \%)$ & \\
\hline Age, median (min-max) & $69(36.5-85.1)$ & & $69.5(43.2-85.1)$ & $67.8(43.8-83.9)$ & $70.9(36.5-84.9)$ & 0.825 \\
\hline \multicolumn{7}{|l|}{ pT stage } \\
\hline $\mathrm{T} 1 \mathrm{a}$ & 38 & 36 & $12(33 \%)$ & $19(53 \%)$ & $5(14 \%)$ & 0.110 \\
\hline $\mathrm{T} 1 \mathrm{~b}$ & 53 & 53 & $29(55 \%)$ & $15(28 \%)$ & $9(17 \%)$ & \\
\hline $\mathrm{T} 2 \mathrm{a}$ & 123 & 120 & $53(44 \%)$ & $49(41 \%)$ & $18(15 \%)$ & \\
\hline $\mathrm{T} 2 \mathrm{~b}$ & 56 & 56 & $23(41 \%)$ & $16(29 \%)$ & $17(30 \%)$ & \\
\hline $\mathrm{T} 3$ & 79 & 78 & $31(40 \%)$ & $29(37 \%)$ & $18(23 \%)$ & \\
\hline $\mathrm{T} 4$ & 29 & 29 & $15(52 \%)$ & $7(24 \%)$ & $7(24 \%)$ & \\
\hline \multicolumn{7}{|l|}{ pN stage } \\
\hline No & 220 & 216 & $94(44 \%)$ & $81(37 \%)$ & $41(19 \%)$ & 0.571 \\
\hline N1 & 116 & 115 & $55(48 \%)$ & $38(33 \%)$ & $22(19 \%)$ & \\
\hline N2 & 42 & 41 & $14(34 \%)$ & $16(39 \%)$ & $11(27 \%)$ & \\
\hline \multicolumn{7}{|l|}{$M$} \\
\hline Mo & 371 & 365 & $160(44 \%)$ & $132(36 \%)$ & $73(20.0 \%)$ & 0.905 \\
\hline M1 & 7 & 7 & $3(43 \%)$ & $3(43 \%)$ & $1(14 \%)$ & \\
\hline \multicolumn{7}{|l|}{ Stage } \\
\hline IA & 69 (18\%) & 68 & $31(46 \%)$ & $26(38 \%)$ & $11(16 \%)$ & 0.703 \\
\hline IB & $80(21 \%)$ & 77 & $32(42 \%)$ & $31(40 \%)$ & $14(18 \%)$ & \\
\hline IIA & $77(20 \%)$ & 77 & $38(49 \%)$ & $29(38 \%)$ & $10(13 \%)$ & \\
\hline IIB & $51(14 \%)$ & 51 & $21(41 \%)$ & $17(33 \%)$ & $13(26 \%)$ & \\
\hline IIIA & $87(23 \%)$ & 85 & $34(40 \%)$ & $27(32 \%)$ & $24(28 \%)$ & \\
\hline IIIB & $7(2 \%)$ & 7 & $4(57 \%)$ & $2(29 \%)$ & $1(14 \%)$ & \\
\hline IV & $7(2 \%)$ & 7 & $3(43 \%)$ & $3(43 \%)$ & $1(14 \%)$ & \\
\hline \multicolumn{7}{|l|}{ Grade } \\
\hline G1 & & 10 & $8(80 \%)$ & $2(20 \%)$ & 0 & 0.073 \\
\hline G2 & & 140 & $58(41 \%)$ & $58(41 \%)$ & $24(17 \%)$ & \\
\hline G3 & & 176 & $73(42 \%)$ & $62(35 \%)$ & $41(23 \%)$ & \\
\hline \multicolumn{7}{|l|}{ Resection } \\
\hline R0 & 330 & 326 & $144(44 \%)$ & 117 (36\%) & $65(20 \%)$ & 0.966 \\
\hline R1 & 45 & 43 & $18(42 \%)$ & $17(39 \%)$ & $8(19 \%)$ & \\
\hline $\mathrm{R} 2$ & 3 & 3 & $1(33 \%)$ & $1(33 \%)$ & $1(33 \%)$ & \\
\hline \multicolumn{7}{|l|}{ Smoking } \\
\hline Pack-years, median (min-max) & $50(2-200)$ & & $50.0(5.0-150.0)$ & $45.0(2.0-200.0)$ & $50.0(16.0-150.0)$ & 0.425 \\
\hline
\end{tabular}

experienced in lung pathology and trained for PD-L1 evaluation (SB). Examples of the staining are shown in Figure 1. The tumor proportion score was assessed using the currently most relevant cutoffs applied in clinical studies (in \%): $<1,1-<50$, and $\geq 50 .^{24}$ Each tissue microarray core was scored separately. A final score was calculated as mean percent across all tissue microarray cores/tumor.

The numbers of $\mathrm{CD}^{+}$and $\mathrm{CD}^{+}$tumor infiltrating lymphocytes were determined using image analysis (Aperio Image Scope), and adjusted for core completeness. The algorithm (provided in the supplementary Table S1) was previously determined by comparison with manual scoring on a training set of 70 cases.

\section{Statistical Analysis}

Statistical analysis was performed using IBM SPSS Statistics 24 (IBM Corporation, Armonk, USA). For group comparisons, crosstabs, $\chi^{2}$-tests, and Fisher's exact tests were performed. For associations between continuous variables, Spearman's rho correlation analysis was used. Associations between immunohistochemical staining patterns and the number of tumor infiltrating lymphocytes were evaluated using the non-parametric Mann-Whitney $U$-test. For survival analysis, disease-specific survival and time to recurrence were assessed instead of overall survival, due to the high number of non-tumorrelated deaths. 

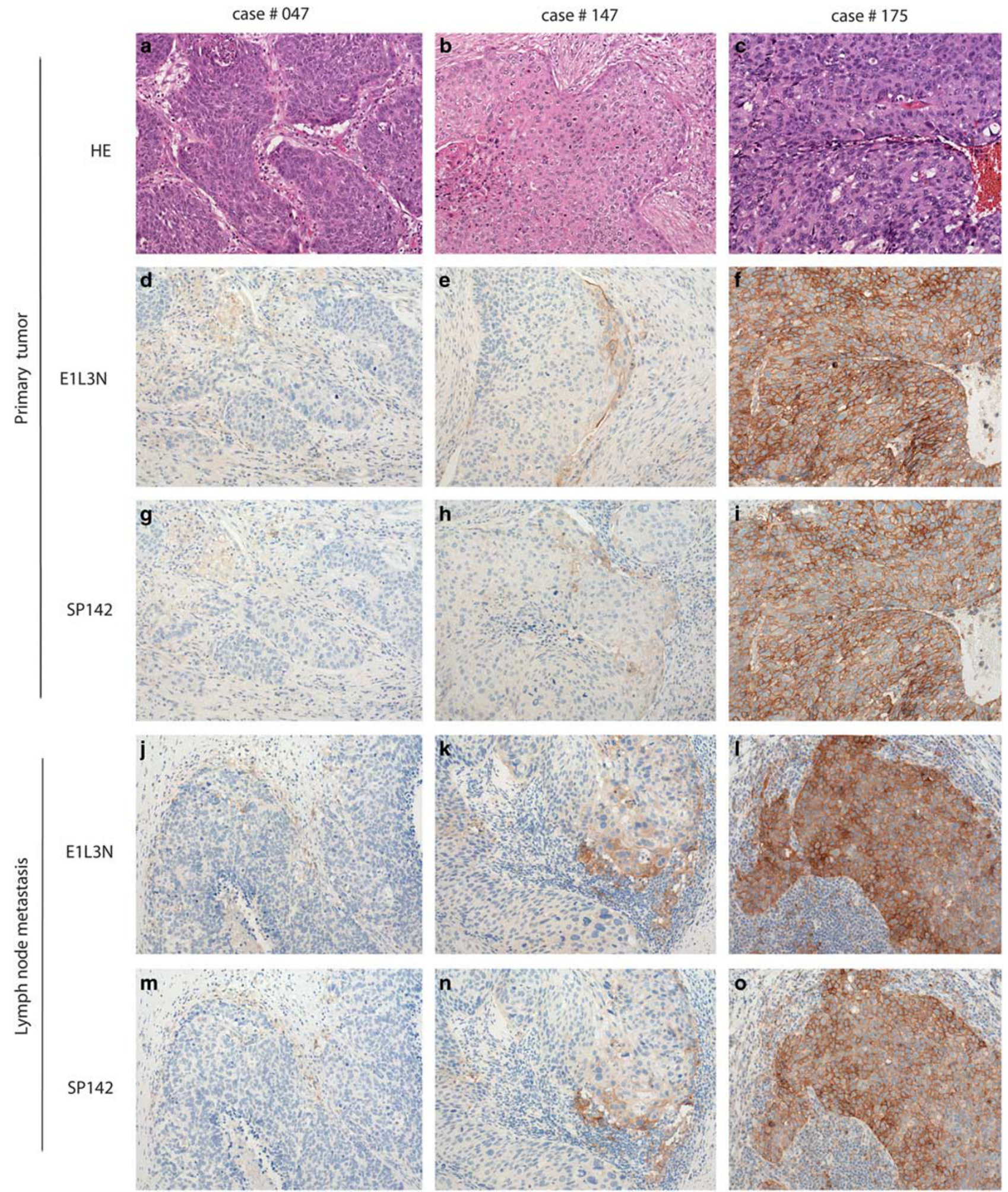

Figure 1 Examples of different increments of membranous expression of PD-L1 using both antibodies in the primary tumors and paired mediastinal lymph node metastases. Primary tumors (a-c, hematoxylin and eosin; $\mathbf{d}-\mathbf{i}$, PD-L1) and lymph node metastases (j-o, PD-L1). Case \#047 (left column): < 1\% PD-L1, case \#147 (middle column): 1-<50\% PD-L1; case \#175 (right column): $\geq 50 \%$ PD-L1. (Total magnification $\times 200)$. 
We determined the prognostically most relevant cutoff of PD-L1 expression using the split-sample validation method, whereby we identified the relevant cutoff on an observation group and validated it on the validation group. For this, the total cohort was divided into two groups of equal size that were homogenized according to UICC/AJCC TNM stage and date of surgery, but otherwise randomly separated (observation group: $N=139$; validation group: $N=139$ ). Kaplan-Meier plots were first generated for the three-tier classification $(<1 \%, 1-<50 \%$, and $\geq 50 \%$ ) and for two groups using a two-tier classification with $50 \%$ cutoff. The prognostically most relevant grouping was then applied for further analysis, including Cox regression analysis for multivariate analysis. $P$-values were two sided and regarded as significant, if $P<0.05$.

\section{Results}

\section{PD-L1 Expression Patterns}

PD-L1 expression was evaluable in 372 (E1L3N) and 370 tumors (SP142). PD-L1 was negative $(<1 \%)$ in 163 (44\%, E1L3N) or 231 tumors (62\%, SP142). Positivity of $1-<50 \%$ was observed in 135 (36\%, E1L3N) or 92 tumors (25\%, SP142) and reached $\geq 50 \%$ in $74(20 \%$, E1L3N) or 47 tumors $(13 \%$, SP142) (Figure 1). The tumor proportion score correlated significantly between the two antibodies $(r=0.781$; $P<0.001$ ), although clone E1L3N showed higher sensitivity, especially in cases with low PD-L1 expression. Detailed values for all increments and comparing both antibody clones are provided in Table 2.

The tumor proportion score correlated significantly between full tissue sections and corresponding tissue microarray cores $(P<0.001$, both antibodies, $N=41$ ), validating our tissue microarraybased approach, with congruence rates of 29/41 (E1L3N and SP142) for the three-tier classification $(<1 \%, 1-<50 \%$, and $\geq 50 \%)$ and $39 / 41$ (E1L3N) and 40/41 (SP142) for the two-tier classification with $50 \%$ cutoff. The discrepant results were predominantly seen within the group of $1-<50 \%$ PD-L1 expression initially assessed $(1-4,5-<9,10-24$, and $25-49 \%$; data not shown), whereas staining patterns were very homogeneous in cases with scores $<1 \%$

Table 2 Correlation of tumoral PD-L1 stainings using clones E1L3N and SP142 for the different increments

\begin{tabular}{|c|c|c|c|c|}
\hline & \multicolumn{3}{|c|}{ PD-L1 (SP142) tumor proportion score } & \multirow{2}{*}{ Total } \\
\hline & $<1 \%$ & $1-<50 \%$ & $\geq 50 \%$ & \\
\hline \multicolumn{5}{|c|}{ PD-L1 (E1L3N) tumor proportion score } \\
\hline$<1 \%$ & 160 & 2 & 0 & 162 \\
\hline $1-<50 \%$ & 69 & 66 & 0 & 135 \\
\hline$\geq 50 \%$ & 2 & 24 & 47 & 73 \\
\hline Total & 231 & 92 & 47 & 370 \\
\hline
\end{tabular}

or $\geq 50 \%$. This was also seen in the whole tissue microarray cohort across the eight cores/tumor: For E1L3N, 127/163 (78\%) cases were negative (ie, $<1 \%$ ) in all cores, and 65/74 (88\%) cases reached $\geq 50 \%$ in all cores. Similar results were seen for SP142, with $197 / 231(85 \%)$ cases homogenous for score $<1 \%$ and $41 / 47(87 \%)$ cases homogenous for score $\geq 50 \%$.

\section{Correlation of PD-L1 Expression with Clinico-Pathological Parameters}

There was an antibody-dependent correlation between higher tumor proportion scores and higher pT categories (three-tier classification; E1L3N: $P=0.11$; SP142: $P=0.032$ ) but not for other clinical and pathological parameters (Table 1). However, higher PD-L1 scores correlated significantly with higher $\mathrm{CD}^{+}$tumor infiltrating lymphocytes count $(P<0.001$ for both antibodies and all increments; Supplementary Figure S1).

\section{Survival Analysis-Observation Group}

In univariate analysis, the following clinico-pathological factors were associated with longer diseasespecific survival and time to recurrence in the observation group: lower UICC/AJCC stage $(P<0.001$ each), complete tumor resection $(P<0.001 ; P=0.15)$, and in trend female gender $(P=0.129 ; P=0.215)$. Tumor grading, patient's age (cutoff: median), and the number of tumor infiltrating lymphocytes assessed as mean over all tissue microarray cores had no prognostic value and were not included in further multivariate analysis nor survival analysis on the validation cohort.

The prognostically relevant cutoff for PD-L1 expression was determined using Kaplan-Meier plots that constantly showed that PD-L1 scores $\geq 50 \%$ were associated with the worst diseasespecific survival and time to recurrence (Figure 2 for E1L3N, Supplementary Figure S2 for SP142). This was seen for both PD-L1 antibodies used for expression analysis, and was also demonstrated by log rank analysis (disease-specific survival: $P=0.035$ for E1L3N and $P=0.002$ for SP142; time to recurrence: $P=0.056$ for E1L3N and $P<0.001$ for SP142). Multivariate analysis showed that PD-L1 expression was an independent prognostic factor for disease-specific survival (E1L3N: HR $=2.768$; 95\% $\mathrm{CI}=1.149-6.666 ; P=0.023$ and SP142: $\mathrm{HR}=4.153$; $95 \% \mathrm{CI}=1.564-11.022 ; P=0.004)$ and time to recurrence (E1L3N: $\mathrm{HR}=2.164 ; 95 \% \mathrm{CI}=1.056-4.436$; $P=0.035$ and SP142: $\mathrm{HR}=3.559 ; 95 \% \mathrm{CI}=1.652-$ 7.668; $P=0.001$ ), besides UICC/AJCC stage (Table 3 for E1L3N and Supplementary Table S2 for SP142).

\section{Survival Analysis_-Validation Group}

Application of the $50 \%$ cutoff on the validation group showed similar prognostic values for PD-L1 

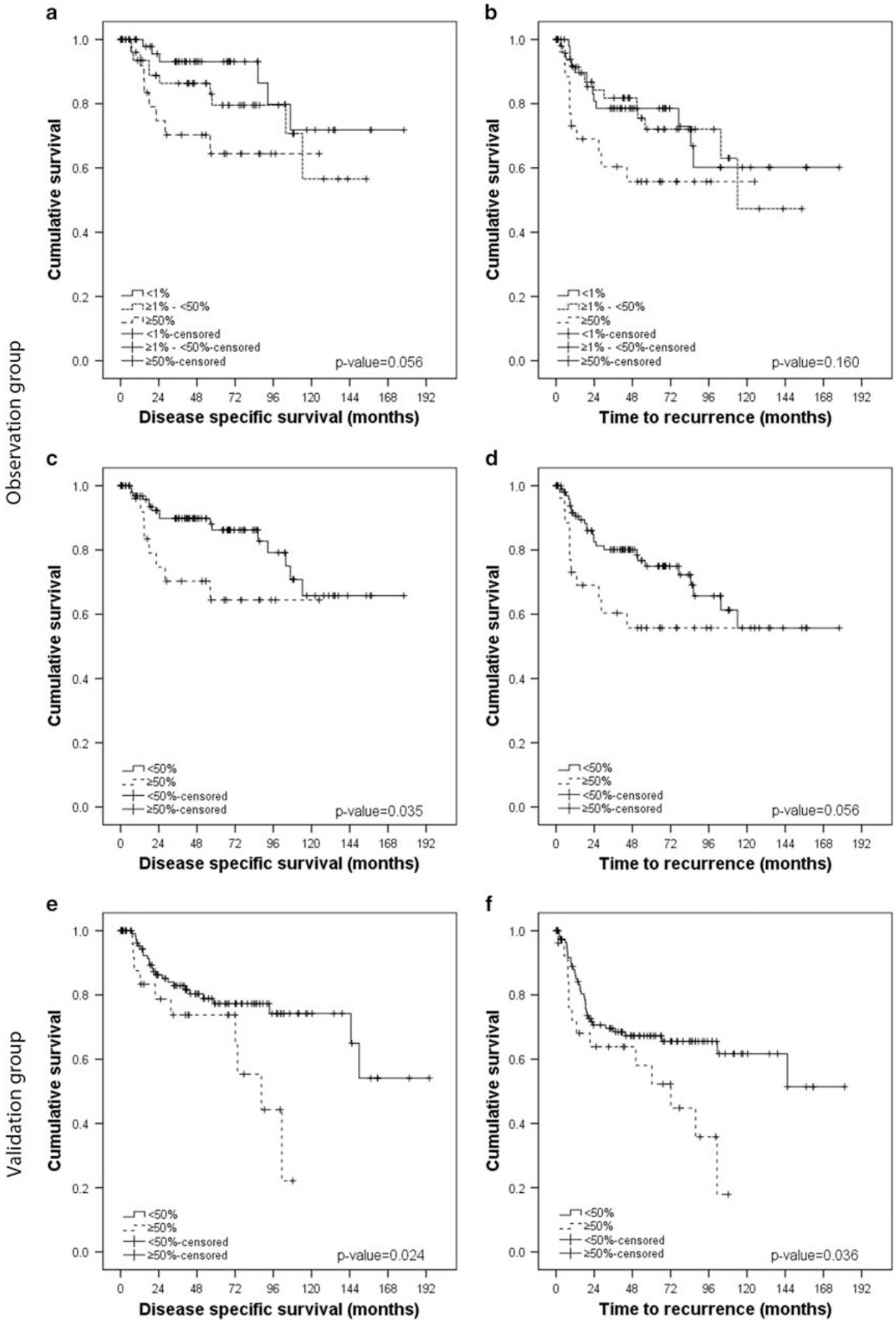

Figure 2 Kaplan-Meier plots for the development (a-d) of the prognostically most relevant cutoff for PD-L1 expression using clone E1L3N in the observation cohort $(N=139$; $\mathbf{a}-\mathbf{b}$, three-tier; $\mathbf{c}-\mathbf{d}$, two-tier) and its application in the validation cohort (e-f, $N=139)$ for diseasespecific survival (DSS) and time to recurrence (TTR). 
Table 3 Multivariate analysis on the observation group (PD-L1 expression using clone E1L3N)

\begin{tabular}{|c|c|c|c|c|}
\hline \multirow{2}{*}{ Parameter } & \multirow{2}{*}{$H R$} & \multicolumn{2}{|c|}{$95.0 \% C I$} & \multirow{2}{*}{$\mathrm{P}$-value } \\
\hline & & Min & $\operatorname{Max}$ & \\
\hline \multicolumn{5}{|l|}{ Disease-specific survival } \\
\hline UICC/AJCC stage & 1.498 & 1.042 & 2.155 & 0.029 \\
\hline Gender & 0.277 & 0.036 & 2.129 & 0.217 \\
\hline $\begin{array}{l}\text { Resection status (complete } \\
\text { versus incomplete) }\end{array}$ & 1.661 & 0.539 & 5.124 & 0.377 \\
\hline PD-L1 (50\% cutoff) & 2.768 & 1.149 & 6.666 & 0.023 \\
\hline \multicolumn{5}{|l|}{ Time to recurrence } \\
\hline UICC/AJCC stage & 1.350 & 1.030 & 1.768 & 0.030 \\
\hline Gender & 0.520 & 0.156 & 1.733 & 0.287 \\
\hline $\begin{array}{l}\text { Resection status (complete } \\
\text { versus incomplete) }\end{array}$ & 1.013 & 0.369 & 2.780 & 0.980 \\
\hline PD-L1 (50\% cutoff) & 2.164 & 1.056 & 4.436 & 0.035 \\
\hline
\end{tabular}

Table 4 Multivariate analysis on the validation group (PD-L1 expression using clone E1L3N)

\begin{tabular}{|c|c|c|c|c|}
\hline \multirow{2}{*}{ Parameter } & \multirow{2}{*}{$H R$} & \multicolumn{2}{|c|}{$95.0 \% C I$} & \multirow{2}{*}{$\mathrm{P}$-value } \\
\hline & & Min & $\operatorname{Max}$ & \\
\hline \multicolumn{5}{|l|}{ Disease-specific survival } \\
\hline UICC/AJCC stage & 1.533 & 1.162 & 2.022 & 0.003 \\
\hline Gender & 0.862 & 0.325 & 2.285 & 0.765 \\
\hline $\begin{array}{l}\text { Resection status (complete } \\
\text { versus incomplete) }\end{array}$ & 1.332 & 0.545 & 3.254 & 0.530 \\
\hline PD-L1 (50\% cutoff) & 1.978 & 0.928 & 4.214 & 0.077 \\
\hline \multicolumn{5}{|l|}{ Time to recurrence } \\
\hline UICC/AJCC stage & 1.432 & 1.164 & 1.761 & 0.001 \\
\hline Gender & 0.813 & 0.377 & 1.753 & 0.597 \\
\hline $\begin{array}{l}\text { Resection status (complete } \\
\text { versus incomplete) }\end{array}$ & 1.272 & 0.612 & 2.647 & 0.519 \\
\hline PD-L1 (50\% cutoff) & 1.571 & 0.838 & 2.944 & 0.159 \\
\hline
\end{tabular}

expression for disease-specific survival $(P=0.024$ for E1L3N and $P=0.101$ for SP142) and time to recurrence $(P=0.036$ for E1L3N and $P=0.247$ for SP142) (Figure 2 for E1L3N and Supplementary Figure S2 for SP142). In multivariate analysis, UICC/AJCC stage was independent prognostic factor for disease-specific survival and time to recurrence. PD-L1 expression $\geq 50 \%$ determined by clone E1L3N did not reach statistical significance but retained a higher HR compared to UICC/AJCC stage (disease-specific survival: $\mathrm{HR}=1.978 ; 95 \% \mathrm{CI}=0.928-4.214 ; P=0.077$ and time to recurrence: $\mathrm{HR}=1.571 ; 95 \% \mathrm{CI}=0.838-2.944$; $P=0.159$; Table 4). PD-L1 expression determined by clone SP142 was not an independent prognostic factor in the validation cohort (Supplementary Table S3).

The Kaplan-Meier plots for the total cohort and the respective multivariate analyses are provided for completeness as Supplementary Material (Supplementary Figure S3 and Supplementary Tables S4 and S5).

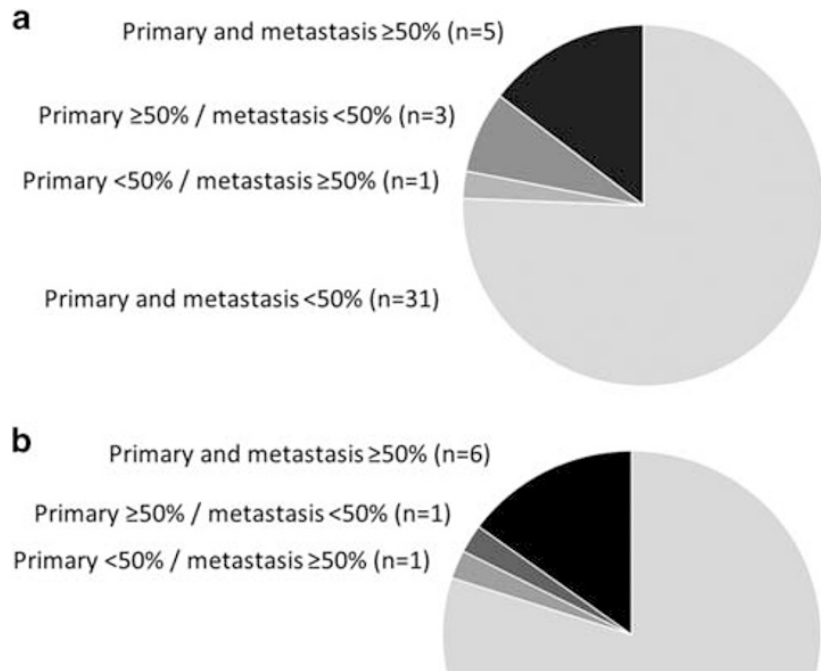

Primary and metastasis $<50 \%(n=32)$

Figure 3 Correlation of PD-L1 expression using the cutoff $50 \%$ (a, clone E1L3N; b, clone SP142) between primary tumors and paired N2 lymph node metastases.

\section{Survival Analysis-Subgroup with Adjuvant Radio- or Chemotherapy}

A subgroup analysis of patients who were treated with adjuvant radio- or chemotherapy according to standard clinical guidelines, taking into account postoperative stage and patients' risk factors ( $N=144$; survival data available for $N=120)$, showed also significant associations of PD-L1 expression with disease-specific survival (E1L3N: $P=0.041$, SP142: $P=0.028$ and in trend for time to recurrence (E1L3N: $P=0.121$, SP142: $P=0.091$ ) (Supplementary Figure S4).

\section{Congruent PD-L1 Expression in N2 Lymph Node Metastases}

Using full-slide sections, PD-L1 scores $\geq 1 \%$ were detected in 19/40 (48\%, E1L3N) or 16/40 (40\%, SP142) N2 lymph node metastases, with score $\geq 50 \%$, in six cases $(15 \%$, E1L3N) and seven cases (18\%, SP142), respectively. As in primary tumors, staining of both antibodies showed a significant correlation $(P<0.001)$ with a complete congruence of $34 / 40(85 \%)$ cases in the three-tier classification and $39 / 40(98 \%)$ cases in the two-tier classification with the $50 \%$ cutoff.

PD-L1 tumor proportion scores correlated significantly between primary tumors and N2 metastases $(P<0.001$ both antibodies $)$. The scores were completely congruent in 24/40 (60\%, E1L3N) and 28/40 cases $(70 \%$, SP142) for the three-tier increments, and $36 / 40(90 \%, \mathrm{E} 1 \mathrm{~L} 3 \mathrm{~N})$ and $38 / 40$ cases (95\%, SP142) for the two-tier increments using the prognostically relevant 50\% cutoff (Figure 3). 


\section{Discussion}

Data regarding the prognostic value of PD-L1 expression in NSCLC are remarkably heterogeneous. As recently reviewed in detail, some studies show an association with worse prognosis, while others report the opposite or no prognostic impact. ${ }^{25,26}$ Discrepancies may be explained by methodological variances, eg, differences in the collectives investigated, which may be quite heterogeneous regarding histology, stage, and ethnicity, confounding factors like previous therapy, staining heterogeneity, and detection methodology. Here we reliably validate the adverse prognostic value of tumoral PD-L1 expression $\geq 50 \%$ in pulmonary squamous cell carcinoma, a cutoff which is relevant for selecting patients for first-line therapy with pembrolizumab. ${ }^{12}$ In our retrospective, single center study on a large and clinically well-annotated European cohort of primary resected squamous cell carcinomas, we have included only true pulmonary squamous cell carcinomas, reliably excluding pulmonary metastases by excluding patients with previous or concomitant squamous cell carcinomas of other sites. Histology was re-evaluated according to current guidelines. ${ }^{2,27}$ Because there is evidence that chemotherapy and radiotherapy may alter PD-L1 expression, we reliably included only therapy-naive tumors. ${ }^{28}$ Patients with pulmonary squamous cell carcinomas often have significant comorbidities, reflected by the high number of non-tumor-associated deaths in our cohort $(63 \%)$. Therefore, we assessed diseasespecific survival and time to recurrence rather than overall survival, showing the independent adverse prognostic value of PD-L1 expression for both parameters. Recent studies suggested that prognostic value of PD-L1 expression may be weakened or increased when adjusting for postoperative therapy, and thus overwhelmed by treatment effect. ${ }^{29} \mathrm{We}$ show that PD-L1 expression remains a prognostic marker for poor outcome in the adjuvantly treated subcohort.

Positivity for PD-L1 has been very heterogeneously defined in the different studies and clinical trials as expression over $1,5,10,25$, or $50 \%{ }^{4-8,24,26,30}$ The cutoffs 1 and $50 \%$ are currently the most relevant ones and show the best reproducibility with regard to the evaluation of the immunohistochemical staining. ${ }^{20}$ Assessing these three increments, we validated the best prognostic discrimination for a PD-L1 tumor proportion score of $\geq 50 \%$, using the split-sample size validation method by dividing our total cohort into the observation group and an internal validation group.

We assessed heterogeneity of PD-L1 staining in the primary by using eight tissue microarray cores/ tumor and comparing the results gained by tissue microarray core scoring with those from full-slide scoring in the N2-positive subcohort. For the clinically and prognostically most significant twotier approach (50\% cutoff), for both antibodies the correlation of full slides with tissue microarray cores reached $>95 \%$ and complete homogeneity over all cores/case was $>87 \%$. This validates our PD-L1 scores assessed on the tissue microarray for the whole cohort and thus our results regarding the prognostic value of PD-L1 expression. On the other hand, by showing homogeneity of less than $100 \%$ over all tissue microarray cores assessed, our data also corroborate a recently published study reporting a sensitivity of a single tissue microarray core for scoring PD-L1 expression $\geq 50 \%$ of around $95 \%$ for squamous cell carcinomas. ${ }^{22}$ The authors conclude that assessing PD-L1 status on a single small sample, as it is done on small biopsies of advanced tumors in clinical routine, may be unreliable. ${ }^{22}$

Immunohistochemical assessment of PD-L1 can be performed using different PD-L1 clones and different detection systems, ${ }^{13}$ and pre-set kits are associated to the different compounds used in clinical studies., $.5,7,31$ Most cross-validation studies compare the commercially available kits. $^{24,32}$ Recently, Adam et $a l^{33}$ compared a large number of kits and laboratorydeveloped tests and presented the feasibility of an equally high sensitivity of PD-L1 staining using clone E1L3N. PD-L1 staining using clone E1L3N has been shown to render equivalent results to DAKO clones 22C3 and 28-8 by others. ${ }^{34}$ Our PD-L1 staining and readout using E1L3N has been duly validated by external quality assessment. Quantification of PD-L1 was performed by light microscopy. A recently published excellent study on NSCLC compared the PD-L1 reading of pathologists with quantitative immunofluorescence using automated morphometric evaluation, and showed a very high concordance of $94 \%$ for tumor cell staining. ${ }^{20}$ Moreover, PD-L1 scores were assessed for each of the tissue microarray cores separately, and the mean value/case was used for further evaluations, denoting an additional level of reliability. Therefore, and given the fact that pathologist-read evaluation of PD-L1 staining was used in the clinical studies and is used in daily routine, we consider our approach as valid and reliable.

In daily clinical settings, only tissue from lymph node metastases may be available for biomarker testing. We focused on mediastinal lymph node metastases $(\mathrm{N} 2)^{18}$ to assure true lymphatic spread, and showed high concordance $(\geq 90 \%)$ between primaries and paired metastases for the two-tier approach ( $50 \%$ cutoff). The congruence seems to be significantly lower in adenocarcinoma, where discrepant results were noted in up to $38 \%$ of paired samples of primaries and lymph node metastases (N1: $N=33, \mathrm{~N} 2: N=32, \mathrm{~N} 3: N=1$ ), presumably due to the heterogeneity of growth patterns. ${ }^{35}$ Lower concordance rates of $70.3 \%$ have also been described in a Korean cohort of pulmonary squamous cell carcinomas and associated pN-unspecified local lymph node metastases, but this may be due to the different cutoff value of $10 \%$ set do define PD-L1 positivity, and assessment in only one tissue microarray core/tumor. ${ }^{30}$ 
$\mathrm{CD}^{+} \mathrm{T}$ cell counts are also in the focus when PDL1 expression is assessed. They are the effector $\mathrm{T}$ cells disabled in their anti-tumoral function by activation of the PD1/PD-L1 pathway, but they also can induce PD-L1 expression through production of interferon-gamma. ${ }^{36}$ Concordant to previous reports, ${ }^{30,35}$ PD-L1 expression and the number of tumor infiltrating lymphocytes correlated positively in our cohort.

In summary, we demonstrated an adverse prognostic value of PD-L1 expression in a clinically wellannotated Western patients' cohort of primary resected pulmonary squamous cell carcinomas. PDL1 expression was highly concordant in primary tumors and N2 lymph node metastases, which may be the only tumor tissue available in clinical settings, implicating they are suitable for assessment of PD-L1 status in pulmonary squamous cell carcinomas. The results of this study add further knowledge to the ardently discussed field of PD-L1 expression in lung cancer.

\section{Acknowledgments}

The authors gratefully acknowledge the Translational Research Unit of the Institute of Pathology for excellent technical support, and the support of the Tissue Bank Bern at the Institute of Pathology, University of Bern, in acquiring patient tissue, and the Cancer registry Bern for support acquiring survival data.

\section{Disclosure/conflict of interest}

SB and RL have conflicts of interest outside the present work: Consultancy honoraria for MSD, Roche, and Bristol-Myers Squibb. All remaining authors have declared no conflicts of interest.

\section{References}

1 Global Burden of Disease Cancer C, Fitzmaurice C, Allen C, et al. Global, regional, and national cancer incidence, mortality, years of life lost, years lived with disability, and disability-adjusted life-years for 32 cancer groups, 1990 to 2015: a systematic analysis for the Global Burden of Disease Study. JAMA Oncol 2016;3:524-548.

2 Novello S, Barlesi F, Califano R, et al. Metastatic nonsmall-cell lung cancer: ESMO Clinical Practice Guidelines for diagnosis, treatment and follow-up. Ann Oncol 2016;27:v1-v27.

3 Langer CJ, Obasaju C, Bunn P, et al. Incremental innovation and progress in advanced squamous cell lung cancer: current status and future impact of treatment. J Thorac Oncol 2016;11:2066-2081.

4 Garon EB, Rizvi NA, Hui R, et al. Pembrolizumab for the treatment of non-small-cell lung cancer. N Engl J Med 2015;372:2018-2028.
5 Fehrenbacher L, Spira A, Ballinger M, et al. Atezolizumab versus docetaxel for patients with previously treated non-small-cell lung cancer (POPLAR): a multicentre, open-label, phase 2 randomised controlled trial. Lancet 2016;387:1837-1846.

6 Herbst RS, Baas P, Kim DW, et al. Pembrolizumab versus docetaxel for previously treated, PD-L1-positive, advanced non-small-cell lung cancer (KEYNOTE-010): a randomised controlled trial. Lancet 2016;387: 1540-1550.

7 Borghaei H, Paz-Ares L, Horn L, et al. Nivolumab versus docetaxel in advanced nonsquamous non-smallcell lung cancer. N Engl J Med 2015;373:1627-1639.

8 Rizvi NA, Mazieres J, Planchard D, et al. Activity and safety of nivolumab, an anti-PD-1 immune checkpoint inhibitor, for patients with advanced, refractory squamous non-small-cell lung cancer (CheckMate 063): a phase 2, single-arm trial. Lancet Oncol 2015;16:257-265.

9 Herbst RS, Soria JC, Kowanetz M, et al. Predictive correlates of response to the anti-PD-L1 antibody MPDL3280A in cancer patients. Nature 2014;515:563-567.

10 Chen DS, Irving BA, Hodi FS. Molecular pathways: next-generation immunotherapy-inhibiting programmed death-ligand 1 and programmed death-1. Clin Cancer Res 2012;18:6580-6587.

11 Du L, Herbst RS, Morgensztern D. Immunotherapy in lung cancer. Hematol Oncol Clin North Am 2017;31: 131-141.

12 Reck M, Rodriguez-Abreu D, Robinson AG, et al. Pembrolizumab versus chemotherapy for PD-L1-positive non-small-cell lung cancer. N Engl J Med 2016;375: 1823-1833.

13 Kerr KM, Nicolson MC. Non-small cell lung cancer, PDL1, and the pathologist. Arch Pathol Lab Med 2016;140: 249-254.

14 Taube JM, Klein A, Brahmer JR, et al. Association of PD-1, PD-1 ligands, and other features of the tumor immune microenvironment with response to anti-PD-1 therapy. Clin Cancer Res 2014;20:5064-5074.

15 Altman DG, McShane LM, Sauerbrei W, et al. Reporting recommendations for tumor marker prognostic studies (REMARK): explanation and elaboration. PLoS Med 2012;9:e1001216.

16 Irmak Y. Construction of a tissue microarray of squamous cell carcinomas of the lung with associated metastases. MM thesis, University of Bern, Bern, 2016.

17 Keller M, Completion of the clinico-pathological parameters und survival data of a collective of squamous cell carcinomas of the lung. MM thesis, University of Bern, Bern, 2017.

18 Sobin LH, Gospodarowicz MK, Christian Wittekind C (eds). International Union Against Cancer (UICC): TNM Classification of Malignant Tumours, 7th edn. WileyBlackwell, 2009, pp 138-146.

19 Zlobec I, Koelzer VH, Dawson H, et al. Next-generation tissue microarray (ngTMA) increases the quality of biomarker studies: an example using CD3, CD8, and CD45RO in the tumor microenvironment of six different solid tumor types. J Transl Med 2013;11:104.

20 Rehman JA, Han G, Carvajal-Hausdorf DE, et al. Quantitative and pathologist-read comparison of the heterogeneity of programmed death-ligand 1 (PD-L1) expression in non-small cell lung cancer. Mod Pathol 2017;30:340-349.

21 Dislich B, Stein A, Seiler CA, et al. Expression patterns of programmed death-ligand 1 in esophageal adenocarcinomas: comparison between primary tumors and 
metastases. Cancer Immunol Immunother 2017;66: 777-786.

22 Gniadek TJ, Li QK, Tully E, et al. Heterogeneous expression of PD-L1 in pulmonary squamous cell carcinoma and adenocarcinoma: implications for assessment by small biopsy. Mod Pathol 2017;30: 530-538.

23 McLaughlin J, Schalper KA, Carvajal-Hausdorf DE, et al. Domain-specific PD-L1 protein measurement in non-small cell lung cancer (NSCLC). J Clin Oncol 2014;32:5s (abstract 8064).

24 Scheel AH, Dietel M, Heukamp LC, et al. Harmonized PD-L1 immunohistochemistry for pulmonary squamouscell and adenocarcinomas. Mod Pathol 2016;29: 1165-1172.

25 Mino-Kenudson M. Programmed cell death ligand-1 (PD-L1) expression by immunohistochemistry: could it be predictive and/or prognostic in non-small cell lung cancer? Cancer Biol Med 2016;13: 157-170.

26 Tsao MS, Le Teuff G, Shepherd FA, et al. PD-L1 protein expression assessed by immunohistochemistry is neither prognostic nor predictive of benefit from adjuvant chemotherapy in resected non-small cell lung cancer. Ann Oncol 2017;28:882-889.

27 Travis WD, Brambilla E, Burke AP, et al. (eds). WHO Classification of Tumours of the Lung, Pleura, Thymus and Heart, 4th edn. International Agency for Research on Cancer (IARC): Lyon, 2015.

28 Champiat S, Ileana E, Giaccone G, et al. Incorporating immune-checkpoint inhibitors into systemic therapy of NSCLC. J Thorac Oncol 2014;9:144-153.
29 Sun JM, Zhou W, Choi YL, et al. Prognostic significance of PD-L1 in patients with non-small cell lung cancer: a large cohort study of surgically resected cases. J Thorac Oncol 2016;11:1003-1011.

30 Kim MY, Koh J, Kim S, et al. Clinicopathological analysis of PD-L1 and PD-L2 expression in pulmonary squamous cell carcinoma: comparison with tumorinfiltrating $\mathrm{T}$ cells and the status of oncogenic drivers. Lung Cancer 2015;88:24-33.

31 Antonia S, Goldberg SB, Balmanoukian A, et al. Safety and antitumour activity of durvalumab plus tremelimumab in non-small cell lung cancer: a multicentre, phase 1b study. Lancet Oncol 2016;17:299-308.

32 Hirsch FR, McElhinny A, Stanforth D, et al. PD-L1 immunohistochemistry assays for lung cancer: results from phase 1 of the "blueprint PD-L1 IHC assay comparison project“. J Thorac Oncol 2016;12:208-222.

33 Adam J, Rouquette I, Damotte D, et al. PL04a.04: Multicentric French Harmonization Study for PD-L1 IHC testing in NSCLC. J Thorac Oncol 2017;12:S11-S12.

34 Rimm DL, Han G, Taube JM, et al. A prospective, multi-institutional, pathologist-based assessment of 4 immunohistochemistry assays for PD-L1 expression in non-small cell lung cancer. JAMA Oncol 2017;3: 1051-1058.

35 Uruga H, Bozkurtlar E, Huynh TG, et al. Programmed cell death ligand (PD-L1) expression in stage II and III lung adenocarcinomas and nodal metastases. J Thorac Oncol 2016;12:458-466.

36 Sanmamed MF, Chen L. Inducible expression of B7-H1 (PD-L1) and its selective role in tumor site immune modulation. Cancer J 2014;20:256-261.

Supplementary Information accompanies the paper on Modern Pathology website (http://www.nature.com/ modpathol) 